\title{
Ultrasound Guided Pectoral Nerve Block Versus Ultrasound Guided Thoracic Paravertebral Block to Supplement General Anesthesia in Simple Mastectomy
}

Mona R. Salem a ${ }^{\text {, Nadia G. El-Sharkawi }}{ }^{\text {, }}$, Inas El-Shazly ${ }^{\text {b }}$, Mohamed A. Mansour ${ }^{\text {b* }}$

${ }^{\mathrm{a}}$ Department of Anesthesia, International Nozha Hospital, Cairo, ${ }^{\mathrm{b}}$ Department of Anesthesia, Intensive Care and Pain Management, Kasr Al Ainy Faculty of Medicine, Cairo University, Cairo, Egypt

* Corresponding author: Mohamed A. Mansour, Email: mohamed.a.mansour77@gmail.com

\begin{abstract}
Background: Breast surgery, can be associated with significant postoperative pain.

Objectives: This study was conducted to compare pectoral nerve block (PECS) and thoracic paravertebral block (TPVB) as supplements to general anesthesia for alleviation of patients' postoperative pain after breast surgery.

Patients and Methods: This prospective cohort study included 60 female patients with ASA I-III, and body mass index < $30 \mathrm{~kg} / \mathrm{m}^{2}$ undergoing mastectomy. Patients were allocated into two equal groups (PECS and TPVB groups). Both types of nerve block were ultrasound-guided. After 30 minutes, general anesthesia was induced. When the mean arterial pressure (MAP) or heart rate (HR) exceeded $20 \%$ of the preoperative value, increments of fentanyl $25 \mu \mathrm{g}$ were given intravenously at 5 minutes intervals. Paracetamol and ketorolac were used for postoperative analgesia. Postoperative pain intensity was measured using visual analogue scale (VAS). The measured parameters included time to the first call for analgesia, intraoperative and postoperative MAP and HR, intraoperative fentanyl consumption, the amount of analgesics used in the first 24 postoperative hours and patients' satisfaction and complications.

Results: The PECS Group showed significantly lower intraoperative fentanyl consumption, longer duration before the first postoperative call for analgesia, lower VAS till 18 hours postoperatively, lower postoperative consumption of both paracetamol and ketorolac, and higher satisfaction score as compared to TPVB group. There were no complications in both groups. Conclusions: Pectoral nerve block was superior to thoracic paravertebral block in terms of intraoperative analgesic consumption, duration of analgesia, postoperative pain score, postoperative analgesic consumption, and patients' satisfaction.
\end{abstract}

Keywords: ultrasound, pectoral, block, paravertebral, mastectomy.

\section{INTRODUCTION}

Breast surgery may be associated with significant postoperative pain ${ }^{(\mathbf{1})}$. Thoracic paravertebral block is known to block pain stimuli from procedures on the thorax that are lateral to the paravertebral muscles such as breast surgery ${ }^{(2)}$.

Recently, pectoral nerve block type I (PECS I) was introduced. It is a kind of superficial nerve block that is easy and reliable. It is directed to both the lateral and medial pectoral nerves through the plane between both pectoralis muscles (the major and the minor) ${ }^{(3)}$. A newer version of pectoral nerve block was recently introduced. It is called "modified PECS block" or type II PECS block. The latter type of block is directed to block the intercostal nerves, which is essential for several types of mastectomies ${ }^{(4)}$.

In the present study, we compared thoracic paravertebral block (TPVB) to pectoral nerve block (PECS) to identify which technique is superior in alleviating patients' postoperative pain after breast surgery.

\section{PATIENTS AND METHODS}

\section{Ethical considerations}

The study was approved by the Ethics Committee, Faculty of Medicine, Cairo University. An informed consent was obtained from each patient before being involved in the study.

\section{Eligibility criteria and study design}

This prospective cohort study was conducted on 60 adult female patients undergoing mastectomy. We included patients 20 to 60 years-old, ASA physical status I to III, with a body mass index less than 30 $\mathrm{kg} / \mathrm{m}^{2}$.

Exclusion criteria: were coagulation or neurological disorders, chronic use of analgesics, infection at the injection site and patient refusal.

Patients were divided into two equal groups according to the type of the block performed; PECS group and TPVB group. Both blocks were ultrasound guided. An intravenous catheter was placed in the contra-lateral upper limb. No premedication was given since full cooperation of patients during the block performance was required. Basic monitoring included ECG, pulse oximetry, and non-invasive blood pressure monitoring.

In PECS group, the patient acquired a supine position with head tilt to the other side of the block. The ipsilateral arm was abducted to 90 degrees. A high frequency linear ultrasound transducer (10-12 MHz) was placed in the infraclavicular region in a parasagittal plane caudal to the lateral third of the 
clavicle to locate the axillary vessels under the pectoralis major and the subclavius muscles. The probe was tilted so that the ultrasound beam was facing medially. The 2 nd rib was recognized under the axillary vessels. The probe was then moved caudally and laterally towards the axilla, and the $3^{\text {rd }}$ rib was identified. At this level, the needle was advanced inplane until the tip lies between pectoralis major and minor muscles and $10 \mathrm{~mL}$ bupivacaine $0.25 \%$ were injected in the fascial plane between the two pectoralis muscles (PECS 1 block). The probe was moved further laterally and the 4th rib was identified. At that level, 20 $\mathrm{ml}$ bupivacaine $0.25 \%$ were injected at the potential space between the lateral extent of pectoralis minor and serratus anterior muscles (PECS 2 block)

In TPVB group, the patient acquired a sitting position. Counting the spinous processes started by $\mathrm{C} 7$, which is the most prominent spinous process. The spine of the scapula corresponds to $\mathrm{T} 3$ spinous process. A high frequency linear ultrasound transducer (10-12 $\mathrm{MHz}$ ) was used to obtain images in the axial (transverse) plane at the level of the 5th thoracic spine, with the transducer positioned just lateral to the spinous process in a transverse and partial oblique position to the vertebral column, parallel to the rib. The probe was moved cranially (approximately $5 \mathrm{~mm}$ ) until an intercostal US view was obtained at the 4th intercostal space. The thoracic paravertebral space (TPVS) appeared as a wedge-shaped hypoechoic layer (black) demarcated by the hyperechoic (bright) reflections of the pleura below and the internal intercostal membrane above. Using an in-plane technique, an 8 -cm, 18-gauge Tuohy needle was inserted from lateral to medial through the external and internal intercostal muscles until the tip of the needle was positioned into the TPVS posterior to the parietal pleura. A pop was often felt as the needle penetrates the internal intercostals membrane. Bupivacaine $0.25 \%$ in a dose of $20 \mathrm{ml}$ was injected. Downward displacement of the pleura was seen with the injection.

Patients were left for 30 minutes to give time for the block to develop and to observe any complications related to it. Induction of general anesthesia was accomplished using fentanyl $2 \mu \mathrm{g} / \mathrm{kg}$, propofol $2-3$ $\mathrm{mg} / \mathrm{kg}$, and rocuronium $0.6 \mathrm{mg} / \mathrm{kg}$. Anesthesia was maintained using isoflurane $1.5 \%$ in $\mathrm{O}_{2}$ /air mixture.

At any time, the mean arterial pressure (MAP) or heart rate (HR) exceeded $20 \%$ of the preoperative value, increments of fentanyl $25 \mu \mathrm{g}$ were given intravenously at 5 minutes intervals till the observed hemodynamic change was corrected. At the completion of surgery, neostigmine and atropine were given for reversal of the muscle relaxation, and the patient was extubated.

\section{Outcome assessment}

Postoperative pain intensity was measured using a visual analog scale (VAS), starting 30 minutes postoperatively; then, repeated every hour till the patient requested analgesia; then, every 2 hours in the first 24 postoperative hours. If VAS was $>3$, the patient was given $1 \mathrm{gm}$ paracetamol infusion over 20 minutes; if pain persisted after $20 \mathrm{~min}$, ketorolac $30 \mathrm{mg}$ intravenously was given. Paracetamol and ketorolac were repeated in the same doses every 6 hours as needed.

The measured parameters included: time to the first call for analgesia after the end of surgery; mean arterial blood pressure and heart rate every $3 \mathrm{~min}$ for the first 30 minutes after injecting the local anesthetic, then every 5 minutes during the surgery; postoperative MAP and HR every hour till 12 hours, then every 2 hours till 24 hours; the total amount of intraoperative fentanyl; the amount of analgesics used in the first 24 postoperative hours; patients' satisfaction with the postoperative analgesia according to a satisfaction score $($ poor $=0$, fair $=1$, good $=2$, excellent $=3$ ), and complications such as intravascular injection, local anesthetic overdose, or pneumothorax.

\section{Ethical approval:}

The study was approved by the Ethics Board of Kasr Al Ainy University and an informed written consent was taken from each participant in the study.

\section{Statistical analysis}

Statistical analysis of data was performed using Paired-Samples T test for numerical variables and ChiSquare test for categorical variables. $\mathrm{P}<0.05$ was considered statistically significant.

\section{RESULTS}

Patients in both groups were of comparable age, weight and ASA physical status as well as procedure time (Table 1).

Table (1): Patients' demographics and procedure time

\begin{tabular}{|l|l|l|l|}
\hline & PECS group & TPVB group & $\mathrm{p}$ \\
\hline Age (year) & $47.2 \pm 7.8$ & $49.7 \pm 7.1$ & 0.19 \\
\hline Weight $(\mathrm{kg})$ & $80.2 \pm 8.3$ & $78.6 \pm 5.3$ & 0.36 \\
\hline $\begin{array}{l}\text { Procedure } \\
\text { time (min) }\end{array}$ & $92.8 \pm 8.9$ & $94.5 \pm 10.1$ & 0.5 \\
\hline
\end{tabular}


After performing the block in the 2 groups, the MAP and the HR were comparable in both groups for 30 minutes. They were comparable in both groups at all the measurement times. The intraoperative fentanyl consumption was significantly lower in PECS group as compared to TPVB group. The patients' request for postoperative analgesia (first postoperative call for analgesia) was after a significantly longer duration in PECS group as compared to TPVB group (Table 2).

Table (2): Intraoperative fentanyl and first postoperative call for analgesia

\begin{tabular}{|l|l|l|l|}
\hline & $\begin{array}{l}\text { PECS } \\
\text { group }\end{array}$ & $\begin{array}{l}\text { TPVB } \\
\text { group }\end{array}$ & $\mathrm{p}$ \\
\hline $\begin{array}{l}\text { Intraoperative } \\
\text { fentanyl }(\mu \mathrm{g})\end{array}$ & $\begin{array}{l}115 \quad \pm \\
18\end{array}$ & $\begin{array}{l}136 \quad \pm \\
24\end{array}$ & $<0.001^{*}$ \\
\hline $\begin{array}{l}\text { First postoperative } \\
\text { call for analgesia } \\
(\mathrm{min})\end{array}$ & $\begin{array}{l}303 \quad \pm \\
15\end{array}$ & $\begin{array}{l}272 \quad \pm \\
40\end{array}$ & $<0.001^{*}$ \\
\hline
\end{tabular}

*Significant

The VAS was significantly lower in PECS group as compared to TPVB group till 18 hours postoperatively; then, it was comparable in the 2 groups between 18 and 24 hours (Table 3 ).

Table (3): Highest VAS during postoperative time periods

\begin{tabular}{|l|l|l|l|}
\hline & $\begin{array}{l}\text { PECS } \\
\text { group }\end{array}$ & $\begin{array}{l}\text { TPVB } \\
\text { group }\end{array}$ & $\mathrm{p}$ \\
\hline $0-4$ hours & $2.5 \pm 0.6$ & $3.4 \pm 1.1$ & $0.001^{*}$ \\
\hline$>4-8$ hours & $2.8 \pm 0.7$ & $3.7 \pm 1.1$ & $0.001^{*}$ \\
\hline$>8-12$ hours & $3.1 \pm 1.0$ & $3.8 \pm 1.3$ & $0.01^{*}$ \\
\hline$>12-18$ hours & $3.2 \pm 1.1$ & $4.1 \pm 1.5$ & $0.01^{*}$ \\
\hline$>18-24$ hours & $3.5 \pm 1.4$ & $3.9 \pm 1.3$ & 0.3 \\
\hline
\end{tabular}

*Significant

Postoperative consumption of both paracetamol and ketorolac was significantly lower in PECS group as compared to TPVB group (Table 4).

Table (4): Postoperative analgesia \& patients' satisfaction

\begin{tabular}{|l|l|l|l|}
\hline & $\begin{array}{l}\text { PECS } \\
\text { group }\end{array}$ & $\begin{array}{l}\text { TPVB } \\
\text { group }\end{array}$ & $\mathrm{p}$ \\
\hline $\begin{array}{l}\text { Paracetamol } \\
(\mathrm{g})\end{array}$ & $2.7 \pm 0.65$ & $3.4 \pm 0.72$ & $<0.001 *$ \\
\hline $\begin{array}{l}\text { Ketorolac } \\
(\mathrm{mg})\end{array}$ & $41 \pm 16.7$ & $54 \pm 25.4$ & $0.02 *$ \\
\hline
\end{tabular}

*Significant

The satisfaction score was significantly higher in PECS group as compared to TPVB group (Figure 1).

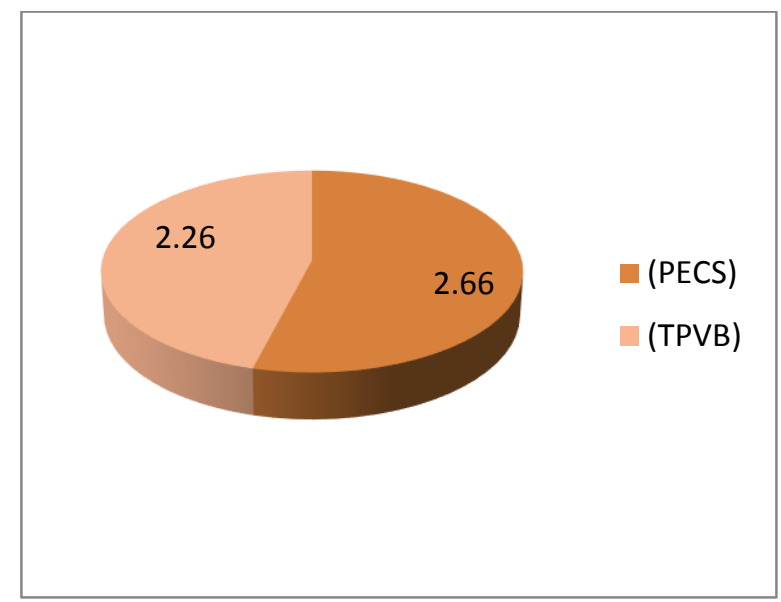

Figure (1): Patients' satisfaction score

There were no recorded minor or major complications in any of the patients during the present study.

\section{DISCUSSION}

Many authors have adopted TPVB as the technique of choice for relief of pain after breast surgery. It has been shown to provide superior analgesia for breast cancer surgery when used in conjunction with general anesthesia ${ }^{(5)}$ and reduces the severity of chronic pain after mastectomy (6). Ultrasound guided TPVB with sedation alone, also is an effective and reliable alternative form of anesthesia for breast surgery when general anesthesia is undesirable or poses an unacceptable risk ${ }^{(7)}$.

Less invasive procedures involving ultrasound guided interfascial injections like the PECS have been suggested as potential analgesic techniques for breast surgery. The PECS I was initially described by Blanco for minor breast surgery like breast expander insertion and subpectoral prosthesis ${ }^{(3)}$. A year later, he described a modified PECS or PECS II for breast surgery involving the axilla as mastectomy, sentinel node dissection, and axillary clearance ${ }^{(4)}$.

The aim of the current study was to compare the efficacy and safety of supplementing general anesthesia with ultrasound guided PECS or ultrasound guided single injection TPVB in female patients undergoing mastectomy.

In previous studies, single injection TPVB has been performed at the level of the 3rd thoracic vertebra ${ }^{(8)}$, 4th thoracic vertebra ${ }^{(9)}$ and 5 th thoracic vertebra (10).

Buckenmaier and his colleagues ${ }^{(11)}$ showed that a block level of T1-T6 provided adequate anesthesia for successful breast surgery with axillary 
clearance. Therefore, in the current study we chose to perform the single injection TPVB at the level of the 4th intercostal space, which is nearly in the middle of the dermatomal area that needs to be blocked.

There were neither intraoperative nor postoperative significant differences in MAP or HR between both groups in the current study.

The intraoperative fentanyl consumption in this study was significantly lower in PECS group (115 $\pm 18 \mu \mathrm{g})$ compared to TPVB group $(136 \pm 24 \mu \mathrm{g})$. This finding is consistent with a previous study of Wahba and Kamal ${ }^{(12)}$ that compared pectoral nerve block with thoracic paravertebral block as supplements to general anesthesia in breast surgery. In that study intraoperative fentanyl consumption was significantly lower $(105 \mu \mathrm{g})$ in pectoral nerve block group as compared to thoracic paravertebral block group (127.5 $\mu \mathrm{g})$. Our results are also in agreement with the study of Bashandy and Abbas ${ }^{(13)}$ where they reported lower intraoperative fentanyl consumption in patients who received general anesthesia and PECS as compared to general anesthesia alone for modified radical mastectomy.

Our results showed that the first postoperative call for analgesia was after a significantly longer duration in PECS group ( $303 \pm 15$ min) compared to TPVB group (272 $\pm 40 \mathrm{~min})$.

This finding is supported by an earlier study that showed a longer latency to the first opioid dose in patients who received single injection paravertebral block as a supplement to general anesthesia as compared to general anesthesia alone ${ }^{(9)}$. Our results agree also with Wahba and Kamal ${ }^{(12)}$ who showed significantly longer time till the first request for analgesia when general anesthesia was supplemented with pectoral nerve block (175 $\mathrm{min})$ as compared to thoracic paravertebral block (137.5 min).

In our study, the VAS was generally low in both groups during the first 24 postoperative hours, with the highest recorded VAS $(4.1 \pm 1.5)$ between 12 18 hours postoperatively. The VAS was significantly lower in PECS group as compared to TPVB group till 18 hours postoperatively; then, it was comparable in both groups between 18 and 24 hours. These results are comparable with other studies on both blocks $(\mathbf{1 , 2 , 8 , 9 , 1 3}$, 14 and 15).

Our results showed low use of postoperative analgesics in both groups, and it was significantly lower in PECS group compared to TPVB group. This finding agrees with previous studies which compared TPVB alone with general anesthesia (9, 14, 16), supplemented general anesthesia with TPVB (8), supplemented TPVB with PECS (17), supplemented general anesthesia with PECS ${ }^{(13)}$, or compared TPVB with PECS ${ }^{(12)}$.

In our study, patients were satisfied with both types of nerve block but the satisfaction score was significantly higher in PECS group $(2.66 \pm 0.47)$ compared to TPVB group $(2.26 \pm 0.73)$. This agrees with previous two studies; a nerve-stimulator guided paravertebral block compared to general anesthesia ${ }^{(14)}$ and a comparison between PECS and TPVB as supplements to general anesthesia ${ }^{(\mathbf{1 2})}$.

Our results showed no complications in any of the patients. This is consistent with previous studies that used TPVB ${ }^{(10,15)}$.

The overall incidence of reported complications with TPVB was between $2.6 \%$ and 5\%; however, the risk of long-term morbidity was extremely low. No fatality directly attributable to TPVB has been reported. The failure rate in experienced hands varies between $6.8 \%$ and $10 \%$. Other specifically reported complications include: hypotension $4.6 \%$, vascular puncture $3.8 \%$, pleural puncture $1.1 \%$, and pneumothorax $0.5 \%{ }^{(18)}$.

PECS I and II were tried as a sole anesthetic in 2 elderly women more than 90 years old. This report was released in 2015 by the Department of Anesthesiology, Nagasaki University School of Medicine, Japan. Case 1 was a 91-year-old female (140 $\mathrm{cm}, 32 \mathrm{~kg}$ ) who presented for simple mastectomy for a large breast tumor. The PECS II was achieved using 35 $\mathrm{ml} 0.2 \%$ ropivacaine. Surgery was completed uneventfully with additional local anesthetic infiltration. Case 2 was a 94-year-old female $(138 \mathrm{~cm}$, $34 \mathrm{~kg}$ ) who presented for lumpectomy of a breast tumor located close to the pectoralis major muscle. The PECS I and II were achieved with a total of $45 \mathrm{ml} 0.2$ $\%$ ropivacaine. Surgery was completed uneventfully (19).

\section{CONCLUSION}

The results of this study showed that pectoral nerve block was superior to thoracic paravertebral block in terms of intraoperative analgesic consumption, duration of analgesia, postoperative pain score, and postoperative analgesic consumption. Patients were more comfortable and satisfied by the pectoral nerve block. Both techniques were safe and there were no complications in either groups. We believe that the use of ultrasound was a cornerstone in the ease and safety of both techniques. We consider both pectoral nerve block and thoracic paravertebral block as quite acceptable supplements to general 
anesthesia during breast surgery. We also highly recommend using pectoral nerve block in future studies.

\section{Conflict of interest:}

The authors have no conflict of interest to declare.

\section{Funding:}

This research did not receive any specific grant from funding agencies in the public, commercial, or notfor-profit sectors.

\section{REFERENCES}

1. Klein SM, Bergh A, Steele SM (2000): Thoracic paravertebral block for breast surgery. Anesth Analg., 90: $1402-5$.

2. Terheggen MK, Wille F, Borel Rinkes IH et al. (2002): Paravertebral blockade for minor breast surgery. Anesth Analg., 94: 355-9.

3. Blanco R (2011): The 'pecs block': a novel technique for providing analgesia after breast surgery. Anaesthesia, 66: 847-8.

4. Blanco R, Fajardo M, Parras M T (2012): Ultrasound description of Pecs II (modified Pecs I): A novel approach to breast surgery. Rev Esp Anestesiol Reanim., 59: 470-5.

5. Schnabel A, Reichl SU, Kranke $P$ et al. (2010): Efficacy and safety of paravertebral blocks in breast surgery: A meta-analysis of randomized controlled trials. Br J Anaesth., 105: 842-52.

6. Karmakar MK, Samy W, Li J (2014): Thoracic paravertebral block and its effects on chronic pain and health-related quality of life after modified radical mastectomy. Reg Anesth Pain Med., 39: 289-98.

7. Simpson J, Ariyarathenam A, Dunn J et al. (2014): Breast Surgery Using Thoracic Paravertebral Blockade and Sedation Alone. Anesthesiol Res Pract., 2014:127467.

8. Kairaluoma PM, Bachmann MS, Korpinen AK et al. (2004): Single-injection paravertebral block before general anesthesia enhances analgesia after breast cancer surgery with and without associated lymph node biopsy. Anesth Analg., 99: 1837-43.
9. Pusch F, Freitag H, Weinstabl C et al. (1999): Singleinjection paravertebral block compared to general anaesthesia in breast surgery. Acta Anaesthesiol Scand., 43: 770-4.

10. Khai DKL (2013): The Use of Single-injection Thoracic Paravertebral Block in Breast Cancer Surgeries in our Asian Population: The Singapore General Hospital Experience. Proc Singap Healthc., 22: 107-13.

11. Buckenmaier CC, Kwon KH, Howard RS et al. (2010): Double-blinded, placebo-controlled, prospective randomized trial evaluating the efficacy of paravertebral block with and without continuous paravertebral block analgesia in outpatient breast cancer surgery. Pain Med., 11: 790-9.

12. Wahba SS, Kamal SM (2014): Thoracic paravertebral block versus pectoral nerve block for analgesia after breast surgery. Egypt J Anaesth., 30: 129-35.

13. Bashandy GM, Abbas DN (2015): Pectoral nerves I and II blocks in multimodal analgesia for breast cancer surgery: a randomized clinical trial. Reg Anesth Pain Med., 40: 68-74.

14. Naja MZ, Ziade MF, Lonnqvist PA (2003): Nervestimulator guided paravertebral blockade vs. general anaesthesia for breast surgery: a prospective randomized trial. Eur J Anaesth., 20: 897-03.

15. Bansal P, Saxena KN, Taneja B et al. (2012): A comparative randomized study of paravertebral block versus wound infiltration of bupivacaine in modified radical mastectomy. J Anaesthesiol Clin Pharmacol., 28: 76-80.

16. Coveney E, Weltz CR, Greengrass R et al. (1998): Use of paravertebral block anesthesia in the surgical management of breast cancer: experience in 156 cases. Ann Surg., 227: 496-01.

17. Sopena-Zubiria LA, Fernández-Meré LA, Valdés Arias C et al. (2012): Thoracic paravertebral block compared to thoracic paravertebral block plus pectoral nerve block in reconstructive breast surgery. Rev Esp Anestesiol Reanim., 59: 12-7.

18. Tighe SQM, Greene MD, Rajadurai N (2010): Continuing Education in Anaesthesia. Crit Care Pain J., 10: 133-7.

19. Murata H, Ichinomiya T, Hara T (2015): Pecs block for anesthesia in breast surgery of the elderly. J Anesth., 29: 644 . 